\title{
Evaluation of a New Brain Tissue Probe for Intracranial Pressure, Temperature and Cerebral Blood Flow Monitoring in Patients with aneurysmal subarachnoid hemorrhage
}

\author{
Seule, M ; Sikorski, C ; Sakowitz, Oliver ; von Campe, G ; Santos, E ; Orakcioglu, B ; Unterberg, A ; \\ Keller, E
}

\begin{abstract}
Objective: To evaluate a brain tissue probe for intracranial pressure (ICP) and temperature (TEMP) monitoring as well as determination of cerebral hemodynamics using a near infrared spectroscopy (NIRS) and indocyanine green (ICG) dye dilution method (NIRS-ICP probe). Methods: The NIRS-ICP probe was applied after aneurysmal subarachnoid hemorrhage if multimodal monitoring was established due to poor neurological condition. ICP and TEMP values were obtained from ventricular catheters and systemic temperature sensors. Repeated NIRS-ICG measurements (2 injections within 30 minutes) were performed daily for determination of cerebral blood flow (CBF), cerebral blood volume (CBV) and mean transit time of ICG (mttICG). Delayed cerebral ischemia was defined as brain tissue oxygen tension $<20 \mathrm{mmHg}$ and/or lactate-pyruvate-ratio $>35$. Results: A total of 128 NIRS-ICG measurements were performed in 10 patients. The correlation coefficient between ICP and TEMP values obtained with the NIRS-ICP probe and values from routine monitoring was $r=0.72$ and $r=0.96$, respectively. The mean value was $30.3 \pm 13.6 \mathrm{ml} / 100 \mathrm{~g} / \mathrm{min}$ for $\mathrm{CBF}, 3.3 \pm 1.2 \mathrm{ml} / 100 \mathrm{~g}$ for CBV, and $6.8 \pm 1.6 \mathrm{sec}$ for mttICG. The coefficient of variation from repeated NIRS-ICG measurements was $10.9 \%$ for CBF, $11.7 \%$ for CBV, and $3.8 \%$ for mttICG. The sensitivity for delayed cerebral ischemia detection was $85 \%$ and the specificity $83 \%$ using a CBFthreshold of $25 \mathrm{ml} / 100 \mathrm{~g} / \mathrm{min}$. Conclusion: Multimodal monitoring using the NIRSICP probe is feasible with high reproducibility of measurement values and the ability to detect delayed cerebral ischemia. No safety concerns exist for the routine clinical use of the NIRS-ICP probe.
\end{abstract}

DOI: https://doi.org/10.1007/s12028-016-0284-4

Posted at the Zurich Open Repository and Archive, University of Zurich

ZORA URL: https://doi.org/10.5167/uzh-126854

Journal Article

Accepted Version

Originally published at:

Seule, M; Sikorski, C; Sakowitz, Oliver; von Campe, G; Santos, E; Orakcioglu, B; Unterberg, A; Keller, E (2016). Evaluation of a New Brain Tissue Probe for Intracranial Pressure, Temperature and Cerebral Blood Flow Monitoring in Patients with aneurysmal subarachnoid hemorrhage. Neurocritical Care, 25(2):193-200.

DOI: https://doi.org/10.1007/s12028-016-0284-4 
EVALUATION OF A NEW BRAIN TISSUE PROBE FOR INTRACRANIAL PRESSURE, TEMPERATURE AND CEREBRAL BLOOD FLOW MONITORING IN PATIENTS WITH ANEURYSMAL SUBARACHNOID HEMORRHAGE

Martin Seule ${ }^{1,2,3}$, Christopher Sikorski ${ }^{1}$, Oliver Sakowitz ${ }^{2}$, Gord vonCampe ${ }^{4}$, Edgar Santos ${ }^{2}$, Berk Orakcioglu ${ }^{2}$, Andreas Unterberg ${ }^{2}$, Emanuela Keller ${ }^{1}$

\footnotetext{
${ }^{1}$ Neurointensive Care Unit, University Hospital and University of Zurich, Switzerland

${ }^{2}$ Department of Neurosurgery, University Heidelberg, Germany

${ }^{3}$ Department of Neurosurgery, Kantonsspital St. Gallen, Switzerland

${ }^{4}$ Department of Neurosurgery, University Graz, Austria
}

\section{CORRESPONDING AUTHOR}

Martin Seule, MD

Department of Neurosurgery, Kantonsspital St. Gallen

Rorschacherstrasse 95, CH - 9007 St. Gallen

Phone: +41-71-494 1886

Fax: +41-71-494 2883

Email: martin.seule@kssg.ch 


\begin{abstract}
Objective: To evaluate a brain tissue probe for intracranial pressure (ICP) and temperature (TEMP) monitoring as well as determination of cerebral hemodynamics using a near infrared spectroscopy (NIRS) and indocyanine green (ICG) dye dilution method (NIRS-ICP probe).

Methods: The NIRS-ICP probe was applied after aneurysmal subarachnoid hemorrhage if multimodal monitoring was established due to poor neurological condition. ICP and TEMP values were obtained from ventricular catheters and systemic temperature sensors. Repeated NIRS-ICG measurements (2 injections within 30 minutes) were performed daily for determination of cerebral blood flow (CBF), cerebral blood volume (CBV) and mean transit time of ICG (mttICG). Delayed cerebral ischemia was defined as brain tissue oxygen tension $<20 \mathrm{mmHg}$ and/or lactate-pyruvate-ratio $>35$.
\end{abstract}

Results: A total of 128 NIRS-ICG measurements were performed in 10 patients. The correlation coefficient between ICP and TEMP values obtained with the NIRS-ICP probe and values from routine monitoring was $r=0.72$ and $r=0.96$, respectively. The mean value was $30.3 \pm 13.6 \mathrm{ml} / 100 \mathrm{~g} / \mathrm{min}$ for $\mathrm{CBF}, 3.3 \pm 1.2 \mathrm{ml} / 100 \mathrm{~g}$ for $\mathrm{CBV}$, and $6.8 \pm 1.6 \mathrm{sec}$ for mttICG. The coefficient of variation from repeated NIRS-ICG measurements was $10.9 \%$ for CBF, $11.7 \%$ for CBV, and $3.8 \%$ for mttICG. The sensitivity for delayed cerebral ischemia detection was $85 \%$ and the specificity $83 \%$ using a CBFthreshold of $25 \mathrm{ml} / 100 \mathrm{~g} / \mathrm{min}$.

Conclusion: Multimodal monitoring using the NIRS-ICP probe is feasible with high reproducibility of measurement values and the ability to detect delayed cerebral ischemia. No safety concerns exist for the routine clinical use of the NIRS-ICP probe.

\title{
KEY WORDS
}

Neuromonitoring; delayed cerebral ischemia; near infrared spectroscopy; indocyanine green; cerebral blood flow; intracranial pressure 


\section{INTRODUCTION}

Intracranial pressure (ICP) monitoring is widely accepted in the management of patients with severe brain injury who are at risk of intracranial hypertension leading to secondary brain damage ${ }^{1}$. However, given the complexity of vasospasm pathophysiology, ICP monitoring alone is insufficient in patients with high-grade aneurysmal subarachnoid hemorrhage (aSAH) ${ }^{2,3}$. In recent years, accumulating clinical evidence suggests that multimodal neuromonitoring including cerebral blood flow (CBF), oxygenation and metabolism can help to avoid delayed cerebral ischemia (DCI) in individual patients ${ }^{4-6}$.

Determination of $\mathrm{CBF}$ is of particular importance within the context of multimodal neuromonitoring, because CBF is the key determinant of cerebral oxygenation and glucose supply. In 2000, thermal diffusion flowmetry for continuous bedside assessment of CBF was introduced and potential clinical benefits were demonstrated in patients with symptomatic vasospasm after aSAH ${ }^{7,8}$. However, the clinical application remains limited because of reduced reliability of CBF measurements in patients with fever and hemodynamic instability ${ }^{9}$.

We tested a new multimodal brain tissue probe for combined ICP and temperature (TEMP) monitoring as well as determination of cerebral hemodynamics and oxygenation (NeMo® Probe and NeMo® System, NeMoDevices AG, Zurich, Switzerland). Relative changes in oxy- and deoxyhemoglobin are provided continuously using fiberoptics for near infrared spectroscopy (NIRS). Additionally, measurements of cerebral hemodynamics are based on a combined NIRS and indocyanine green (ICG) dye dilution mode ${ }^{10}$. After intravenous injection of ICG, quantitative assessment of the mean transit time of the dye (mttICG), cerebral blood volume (CBV) and CBF is provided at the patients' bedside. The safety and proof of concept has been documented in experimental studies and preliminary case reports ${ }^{11-15}$.

The new combined NIRS-ICP probe may offer enhanced modality modes without an additional surgical procedure in patients if ICP monitoring is indicated. The aim of this study was to evaluate the reproducibility of measurement values using the NIRS-ICP probe and the ability to detect DCI in patients with aSAH.

\section{CLINICAL MATERIAL AND METHODS}

This prospective observational study was conducted at the University Zurich and University Heidelberg. Approval was obtained from local institutional review boards and national regulatory authorities (Swiss Agency for Therapeutic Products and German Federal Institute for Drugs and Medical Devices). All patients or their legal representatives gave written informed consent. 


\section{Study Population and Treatment}

We enrolled patients with severe aSAH who had been admitted to the neurointensive care unit after uneventful aneurysm treatment. If continued sedation was necessary due to poor neurological condition, multimodal neuromonitoring (ICP, brain tissue oxygen tension $\left(\mathrm{ptiO}_{2}\right)$, and microdialysis) were established for at least 72 hours. Patients with coagulopathy and/or known allergic reaction against contrast agents containing iodine or ICG were excluded. Other exclusion criteria were age $<18$ or $>75$ years, thyroid disease causing hyperthyroidism, significant kidney and/or liver disease, and a known or potential pregnancy. All patients remained intubated and artificially ventilated and intraventricular catheters were implanted due to acute hydrocephalus (Bactiseal® EVD Catheter, CODMAN, Johnson \& Johnson, Raynham, USA). Temperature (TEMP) was measured using catheter-tipped sensors in the arterial line or urinary bladder. Intracranial hypertension and hemodynamic relevant vasospasm were treated according to recommendations from the Neurocritical Care Society ${ }^{16}$. There were no study specific protocols for any interventions. Therapeutic decisions were not based on results obtained by the NIRS-ICP probe.

\section{Multimodal Neuromonitoring using the NIRS-ICP probe}

The NIRS-ICP probe (NeMo® Probe, NeMoDevices AG, Zurich, Switzerland) is a parenchymal probe with sensors for ICP and TEMP monitoring additionally supplied with optical fibers for NIRS, providing continuous measurements of oxy- and deoxyhemoglobin (Figure 1). A combined NIRS and ICG dye dilution mode was applied for quantitative assessment of CBF, CBV and mttICG ${ }^{10,11}$. With NIRS-ICG dilution, ICG (ICG-Pulsion®, PULSION Medical Systems, Munich, Germany) in a dose of $0.3 \mathrm{mg} / \mathrm{kg}$ body weight was injected into a central venous line followed by the injection of a $10 \mathrm{ml}$ 5\% glucose flush. For implantation of the probe, a bolt (IM3_EU, Licox, Integra LifeScience, Plainsboro, USA) was inserted through a frontal burr hole placed $30 \mathrm{~mm}$ lateral to the midline. The probe was inserted through the bolt, placed into the subcortical white matter ( $25 \mathrm{~mm}$ below the dura mater) and secured by tightening the bolt adapter. The probe position was verified by a post-insertion computed tomography (CT) scan.

\section{Monitoring of Brain Tissue Oxygen and Metabolism}

Monitoring cerebral oxygenation and metabolism was performed in all patients. $\mathrm{PtiO}_{2}$ was measured using a Clark type electrode (CC1.SB, Licox, Integra LifeScience, Plainsboro, USA) and extracellular concentrations of energy metabolism (glucose, lactate, pyruvate) were analyzed using cerebral microdialysis (CMA 70, CMA Mikrodialysis, Solna, Sweden). Both probes were inserted parallel to the NIRS-ICP probe using the same bolt and secured using the Luer lock connector. 


\section{Definition of DCI}

Because all patients in this study were comatose and/or sedated, the definition of DCI was based on findings of $\mathrm{ptiO}_{2}$ measurements and microdialysis as previously reported ${ }^{5,6}$. Thus DCI was defined as cerebral hypoxia $\left(\mathrm{ptiO}_{2}<20 \mathrm{mmHg}\right.$ ) and/or metabolic compromise (glucose level $<1.0 \mathrm{mmol} / \mathrm{l}$ and lactate/pyruvate ratio $>35$ ), indicating secondary ischemia with increased risk of further brain damage.

\section{Study Protocol}

Following aneurysm clipping or coiling, the NIRS-ICP probe was inserted ipsilateral to the hemisphere with the highest risk to develop secondary ischemia. Post-insertion CT scan verified probe location. Repeated NIRS-ICG dye dilution measurements (2 ICG injections within 20-30 minutes), providing absolute values of $\mathrm{CBF}, \mathrm{CBV}$ and $\mathrm{mttICG}$, were performed daily during an observation period of up to 8 days. Measurement values of ICP, TEMP, $\mathrm{ptiO}_{2}$, extracellular glucose and lactate/pyruvate ratio were recorded in parallel to each dye dilution measurement.

\section{$\underline{\text { Statistical Analysis }}$}

Statistical analysis was performed using Excel® (Version 14.5.5, Microsoft ${ }^{\circledR}$, USA) and Prism Graph software (Prism 5.0c, GraphPad Software, Inc., USA). Results are presented as mean \pm standard deviation unless otherwise indicated. Physiological parameters were compared using the MannWhitney test. A p-value $<0.05$ was considered statistical significant. The accuracy of ICP and TEMP values was assessed using Spearman correlation and Bland-Altman plot. The mean difference (bias) and $95 \%$ confidence interval (limits of agreement) were calculated by plotting the difference against their mean ${ }^{17}$. The reproducibility of NIRS-ICG measurements was evaluated using the coefficient of variation from repeated measurements, which was calculated as the ratio of the standard deviation to the mean (coefficient of variation $=$ standard deviation $/$ mean) ${ }^{18}$. The ability to predict DCI was analysed using ROC curves representing the combination of the sensitivity (true positive rate) and specificity (true negative rate) for each possible diagnostic threshold of CBF, CBV and mttICG. The ability to detect DCI was present if the area under the curve and the lower bound of a $95 \%$ confidence interval was $>0.5^{19}$.

\section{RESULTS}

Study Population

Ten patients with a mean age of $47.2 \pm 9.4$ years were included in the study. All aneurysms were located in the anterior circulation, of which 4 were treated with clipping, 4 with coiling and 2 with clipping after incomplete coiling. The NIRS-ICP probe was implanted ipsilateral to the aneurysm in 9 patients and bilaterally in 1 patient. The day of probe insertion was on average $3.3 \pm 2.1$ days after aSAH. A total of 128 ICG injections were performed during an average monitoring period of $6.4 \pm$ 
1.9 days. The average number of ICG injections per patient was $13.3 \pm 3.2$. There were no complications related to probe implantation and/or NIRS-ICG dye dilution measurements.

\section{Accuracy of Intracranial Pressure and Brain Temperature Monitoring}

A total of 42-paired ICP and 35-paired TEMP values were analyzed. Mean $\operatorname{ICP}_{\mathrm{NeMo}}$ and $\operatorname{ICP}_{\text {Control }}(14.8$ $\pm 6.4 \mathrm{mmHg}$ versus $12.8 \pm 4.5 \mathrm{mmHg} ; \mathrm{p}=0.147)$ as well as $\mathrm{TEMP}_{\text {NeMo }}$ and $\mathrm{TEMP}_{\text {Control }}\left(35.1 \pm 1.7^{\circ} \mathrm{C}\right.$ versus $\left.35.6 \pm 1.6{ }^{\circ} \mathrm{C} ; \mathrm{p}=0.165\right)$ did not differ significantly. The scatter and Bland-Altman plots are displayed in Figure 2. There was a good correlation between $\mathrm{ICP}_{\mathrm{NeMo}}$ and $\mathrm{ICP}_{\mathrm{Control}}(\mathrm{r}=0.72,95 \% \mathrm{CI}$ 0.53-0.84; $\mathrm{p}<0.0001)$. The mean bias of all ICP measurements was $2.1 \mathrm{mmHg}$ with limits of agreement of -6.9 to $11.0 \mathrm{mmHg}$. A strong correlation was found between $\mathrm{TEMP}_{\mathrm{NeMo}}$ and $\mathrm{TEMP}_{\mathrm{Control}}$ $(\mathrm{r}=0.96,95 \%$ CI $0.93-0.98 ; \mathrm{p}<0.0001)$ with a mean bias of $0.01{ }^{\circ} \mathrm{C}$ and limits of agreement of -0.5 to $0.5^{\circ} \mathrm{C}$.

\section{Reproducibility of repeated measurements using the NIRS-ICG dye dilution method}

Estimation of cerebral hemodynamics was feasible in 122 of 128 NIRS-ICG measurements. Measurement values were not available after 6 ICG injections due to artifacts or insufficient signal to noise ratio. The mean value was $30.3 \pm 13.6 \mathrm{ml} / 100 \mathrm{~g} / \mathrm{min}$ for $\mathrm{CBF}, 3.3 \pm 1.2 \mathrm{ml} / 100 \mathrm{~g}$ for $\mathrm{CBV}$, and $6.8 \pm 1.6 \mathrm{sec}$ for mttICG. The reproducibility of measurement values was analyzed using the coefficient of variation from 59-pairs of repeated NIRS-ICG measurements. Due to missing values the coefficient of variation could not be calculated in 5-pairs of repeated NIRS-ICG measurements. The observed range of CBF, CBV, and mttICG values is displayed in Table 1. The reproducibility of repeated NIRS-ICG measurements was high with a mean coefficient of variation of $10.9 \%$ for CBF, $11.7 \%$ for CBV, and $3.8 \%$ for mttICG.

\section{Detection of Delayed Cerebral Ischemia}

The diagnosis of DCI was established in 7 patients. A total of 52 of 122 NIRS-ICG measurements were performed with DCI. The values for CBF, CBV, and mttICG are presented in Table 2. Both CBF and CBV were significantly reduced, whereas mttICG increased with DCI. Figure 3 shows the ROC curve generated for each possible diagnostic cutoff. CBF measurements showed the largest area under the curve and proved to be superior to the information obtained by CBV and mttICG alone. The data used for prediction of DCI with respect to a chosen cutoff value is presented in Table 3.

\section{DISCUSSION}

The preliminary results demonstrate that multimodal neuromonitoring using the NIRS-ICP probe is feasible and safe. ICP and TEMP values obtained with the new probe agreed well with values obtained from ventricular drainages and systemic temperature sensors. In addition, repeated NIRS- 
ICG dye dilution measurements provided reliable values for CBF, CBV and mttICG with a high reproducibility under stable clinical conditions. Finally, we demonstrated that CBF measurements predict DCI with acceptable sensitivity and specificity.

The ICP sensor of the NeMo® Probe is based on strain gauge principles of pressure transformation and has been validated against intraventricular pressure monitoring through a fluid-filled catheter connected to an external transducer. The results demonstrated sufficient agreement with a mean difference of $2.1 \mathrm{mmHg}$. The Bland Altman plot (with several data points above the line of identity) suggests a certain degree of overestimation of ICP values measured with the NeMo® Probe. This might be explained on one hand by pathophysiological principles, since the local information provided by intraparenchymal sensors may differ from ICP values obtained by intraventricular catheters ${ }^{20}$. On the other hand, drift over time is a known restriction from intraparenchymal sensors based on strain gauge principles, which might have further contributed to the observed differences ${ }^{21}$. In the context of these well-known limitations of intraparenchymal ICP monitoring, the observed differences were within acceptable limits ${ }^{22}$. TEMP measurements using the NIRS-ICP probe were interchangeable with TEMP values obtained from systemic temperature sensors.

Thermal diffusion flowmetry is so far the only technique, which allows for CBF monitoring in a feasible and user-friendly way directly at the bedside and there is evidence that symptomatic vasospasm after aSAH can be detected by the method ${ }^{7,8}$. However, its clinical application is limited because measurement drifts have been reported over the 30-minute measurement cycle and between automatically performed recalibrations ${ }^{23}$. In contrast to the thermal diffusion probe facilitating continuous CBF measurements, the NIRS-ICP probe provides serial measurements of CBF, CBV and mttICG based on NIRS and the intravenous injection of ICG. The results of this study confirm the reproducibility of measurement values with a standard deviation of $12.9 \mathrm{ml} / 100 \mathrm{~g} / \mathrm{min}$ and a coefficient of variation of $10.9 \%$ for CBF. Kety and Schmidt reported a standard deviation of 12 $\mathrm{ml} / 100 \mathrm{~g} / \mathrm{min}$ from repeated CBF measurements using the nitrous oxide method ${ }^{24}$. Olesen et al. reported a coefficient of variation of $8.2 \%$ from repeated CBF measurements using the xenon dilution method ${ }^{25}$. These findings confirm that the reliability of the NIRS-ICP probe is comparable to previously reported bedside CBF monitoring systems being acceptable for clinical decision-making.

The detection of DCI in comatose and/or sedated patients is based on bedside monitoring systems for determination of cerebral hemodynamics and oxygenation ${ }^{1}$. Transcranial Doppler sonography has been shown to predict symptomatic vasospasm in the middle cerebral artery with good sensitivity (64\%) and specificity (78\%), but was less accurate in predicting anterior cerebral artery vasospasm (sensitivity $45 \%$ and specificity $84 \%)^{26}$. Besides transcranial Doppler sonography, $\mathrm{ptiO}_{2}$ monitoring and cerebral microdialysis are widely accepted as diagnostic tool for early detection of DCI ${ }^{5,6}$. In this 
study, we compared CBF, CBV and mttICG measurements obtained by the NIRS-ICP probe with and without DCI. The reliable detection of DCI using CBF measurements obtained by the NIRS-ICP probe has been demonstrated with a sensitivity of $85 \%$ and specificity of $83 \%$ using a CBF-threshold of $25.0 \mathrm{ml} / 100 \mathrm{~g} / \mathrm{min}$.

A major limitation of the study is the lack of a gold standard for CBF measurements such as xenonenhanced CT as a reference method. Another limitation is that our data rely on a limited number of patients and thus results of this study need to be interpreted as preliminary. Furthermore, the NIRSICP probe provides focal values and DCI may be missed if it occurs besides the tissue illuminated by the probe. Theoretical examinations indicate that the measurement volume of the NIRS-ICP probe is approximately $1 \mathrm{~cm}^{3}$. Finally, it is important to note that continuous NIRS monitoring of cerebral oxygenation was not part of this study. Total hemoglobin concentrations (Hbtotal $=$ oxyhemoglobin + deoxyhemoglobin) reflect $\mathrm{CBV}$, and therefore, can be used for decision-making on when to perform NIRS-ICG dye dilution measurements in daily clinical practice.

\section{CONCLUSION}

In conclusion, the NIRS-ICP probe offers the benefit of multimodal monitoring of ICP, TEMP, CBF, CBV and oximetry parameters with one single brain tissue probe and no additional surgical risk if ICP monitoring is indicated anyway. The new monitoring system might be especially helpful in detection of DCI in patients with aSAH. The accuracy of absolute CBF values have to be further validated in future investigations using imaging studies such as xenon-enhanced or perfusion CT. 


\section{ACKNOWLEDGEMENT}

The authors gratefully thank Dr. Rafael Sauter (Clinical Trial Unit, Kantonsspital St. Gallen, Switzerland) for his statistical support.

\section{SOURCE OF FUNDING}

M. Seule received grant support by the Stiefel-Zangger Foundation of the University Zurich, Switzerland. The project is supported by the Swiss Innovation Promotion Agency (project no. 13553.1PFFLM-LS), Eurostars (project no. E!6526 OPTO-BRAIN), as well as by the Velux and von Tobel foundations. NeMoDevices AG (Zurich, Switzerland) provided technical equipment for this clinical trial.

\section{DISCLOSURE}

Prof. E. Keller has a financial interest in this work as founder and shareholder of NeMoDevices AG, Zurich, Switzerland. 


\section{REFERENCES}

1. Le Roux P, Menon DK, Citerio G, et al. Consensus summary statement of the International Multidisciplinary Consensus Conference on Multimodality Monitoring in Neurocritical Care: a statement for healthcare professionals from the Neurocritical Care Society and the European Society of Intensive Care Medicine. Neurocrit Care 2014;21 Suppl 2:S1-26.

2. Pluta RM, Hansen-Schwartz J, Dreier J, et al. Cerebral vasospasm following subarachnoid hemorrhage: time for a new world of thought. Neurol Res 2009;31:151-8.

3. Macdonald RL, Pluta RM, Zhang JH. Cerebral vasospasm after subarachnoid hemorrhage: the emerging revolution. Nat Clin Pract Neurol 2007;3:256-63.

4. Seule M, Muroi C, Sikorski C, Keller E. Monitoring of cerebral hemodynamics and oxygenation to detect delayed ischemic neurological deficit after aneurysmal subarachnoid hemorrhage. Acta neurochirurgica Supplement 2013;115:57-61.

5. Oddo M, Bosel J. Monitoring of brain and systemic oxygenation in neurocritical care patients. Neurocrit Care 2014;21 Suppl 2:S103-20.

6. Hutchinson P, O'Phelan K. International multidisciplinary consensus conference on multimodality monitoring: cerebral metabolism. Neurocrit Care 2014;21 Suppl 2:S148-58.

7. Vajkoczy P, Roth H, Horn P, et al. Continuous monitoring of regional cerebral blood flow: experimental and clinical validation of a novel thermal diffusion microprobe. $\mathrm{J}$ Neurosurg 2000;93:265-74.

8. Vajkoczy P, Horn P, Thome C, Munch E, Schmiedek P. Regional cerebral blood flow monitoring in the diagnosis of delayed ischemia following aneurysmal subarachnoid hemorrhage. $\mathrm{J}$ Neurosurg 2003;98:1227-34.

9. Jaeger M, Soehle M, Schuhmann MU, Winkler D, Meixensberger J. Correlation of continuously monitored regional cerebral blood flow and brain tissue oxygen. Acta Neurochir (Wien) 2005;147:51-6; discussion 6.

10. Keller E, Nadler A, Alkadhi H, Kollias SS, Yonekawa Y, Niederer P. Noninvasive measurement of regional cerebral blood flow and regional cerebral blood volume by near-infrared spectroscopy and indocyanine green dye dilution. Neuroimage 2003;20:828-39.

11. Keller E, Froehlich J, Muroi C, Sikorski C, Muser M. Neuromonitoring in intensive care: a new brain tissue probe for combined monitoring of intracranial pressure (ICP) cerebral blood flow (CBF) and oxygenation. Acta neurochirurgica Supplement 2011;110:217-20. 
12. Seule M, Keller E, Unterberg A, Sakowitz O. The Hemodynamic Response of Spreading Depolarization Observed by Near Infrared Spectroscopy After Aneurysmal Subarachnoid Hemorrhage. Neurocrit Care 2015;23:108-12.

13. Keller E, Ishihara $\mathrm{H}$, Nadler A, et al. Evaluation of brain toxicity following near infrared light exposure after indocyanine green dye injection. J Neurosci Methods 2002;117:23-31.

14. Keller E, Wolf M, Martin M, Yonekawa Y. Estimation of cerebral oxygenation and hemodynamics in cerebral vasospasm using indocyaningreen dye dilution and near infrared spectroscopy: a case report. J Neurosurg Anesthesiol 2001;13:43-8.

15. Keller E, Nadler A, Niederer P, Yonekawa Y, Imhof HG. A new subdural probe for combined intracranial pressure (ICP) and cerebral blood flow (CBF) monitoring. Acta Neurochir (Wien) 2003;145:1111-5; discussion 5.

16. Diringer MN, Bleck TP, Claude Hemphill J, 3rd, et al. Critical care management of patients following aneurysmal subarachnoid hemorrhage: recommendations from the Neurocritical Care Society's Multidisciplinary Consensus Conference. Neurocritical care 2011;15:211-40.

17. Bland JM, Altman DG. Statistical methods for assessing agreement between two methods of clinical measurement. Lancet 1986;1:307-10.

18. Everitt B. The Cambridge dictionary of statistics. Cambridge, UK ; New York: Cambridge University Press; 1998.

19. Gengsheng Q, Hotilovac L. Comparison of non-parametric confidence intervals for the area under the ROC curve of a continuous-scale diagnostic test. Statistical methods in medical research 2008;17:207-21.

20. Sahuquillo J, Poca MA, Arribas M, Garnacho A, Rubio E. Interhemispheric supratentorial intracranial pressure gradients in head-injured patients: are they clinically important? J Neurosurg 1999;90:16-26.

21. Zhong J, Dujovny M, Park HK, Perez E, Perlin AR, Diaz FG. Advances in ICP monitoring techniques. Neurol Res 2003;25:339-50.

22. Morgalla MH, Krasznai L, Dietz K, Mettenleiter H, Deininger M, Grote EH. Methods of experimental and clinical assessment of the relative measurement accuracy of an intracranial pressure transducer. Technical note. J Neurosurg 2001;95:529-32.

23. Wolf S, Vajkoczy P, Dengler J, Schurer L, Horn P. Drift of the Bowman Hemedex(R) cerebral blood flow monitor between calibration cycles. Acta Neurochir Suppl 2012;114:187-90. 
24. Kety SS, Schmidt CF. The Nitrous Oxide Method for the Quantitative Determination of Cerebral Blood Flow in Man: Theory, Procedure and Normal Values. The Journal of clinical investigation 1948;27:476-83.

25. Olesen J, Paulson OB, Lassen NA. Regional cerebral blood flow in man determined by the initial slope of the clearance of intra-arterially injected 133Xe. Stroke 1971;2:519-40.

26. Suarez JI, Qureshi AI, Yahia AB, et al. Symptomatic vasospasm diagnosis after subarachnoid hemorrhage: evaluation of transcranial Doppler ultrasound and cerebral angiography as related to compromised vascular distribution. Crit Care Med 2002;30:1348-55. 


\section{FIGURE LEGENDS}

Figure 1: A) The NeMo ${ }^{\circledR}$ Probe showing the intracranial pressure and temperature (1), electrical (2), and fiberoptic cable (3) ending in a single wire containing the light emitter (I) and detector (II) as well as the sensor for intracranial pressure (III). B) The NeMo® Control Unit containing the light source and detector electronics. C) A personal computer containing the NeMo® Monitor software for determination of cerebral hemodynamics. D) Intubated and sedated patient with bilateral multimodal neuromonitoring including the $\mathrm{NeMo}{ }^{\circledR}$ Probe $(\boldsymbol{\uparrow} \uparrow)$, brain tissue oxygen monitoring $(\boldsymbol{\uparrow})$ and microdialysis $(*)$.
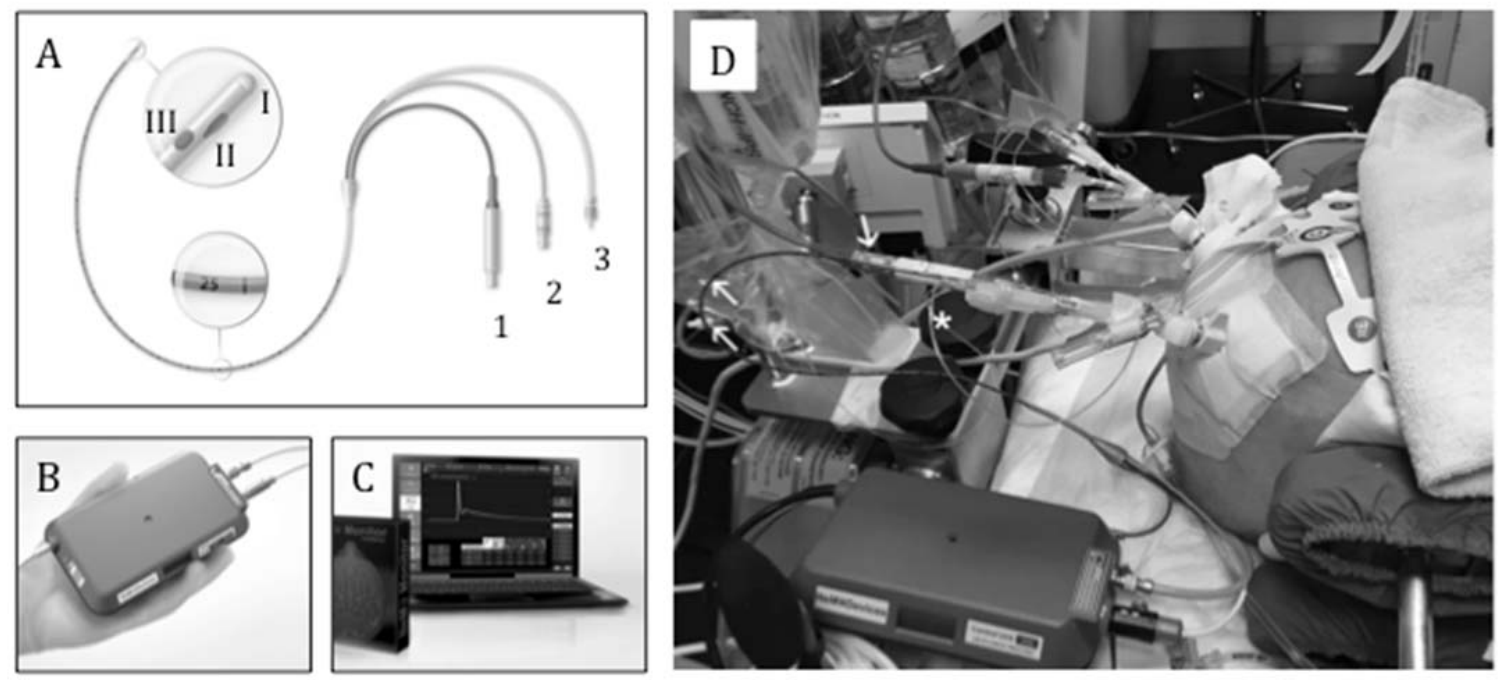
Figure 2: Accuracy of intracranial pressure (ICP) and temperature (TEMP) measurements obtained by the NIRS-ICP probe. A) Correlation between ICP values obtained with the NIRS-ICP probe and intraventricular catheters. B) Bland Altman plot with the difference between ICP values plotted against their mean. C) Correlation between TEMP values obtained with the NIRS-ICP probe and systemic temperature sensors. B) Bland Altman plot with the difference between TEMP values plotted against their mean. Note $-\mathrm{x}=\mathrm{y}$, line of identity; CI confidence interval

A

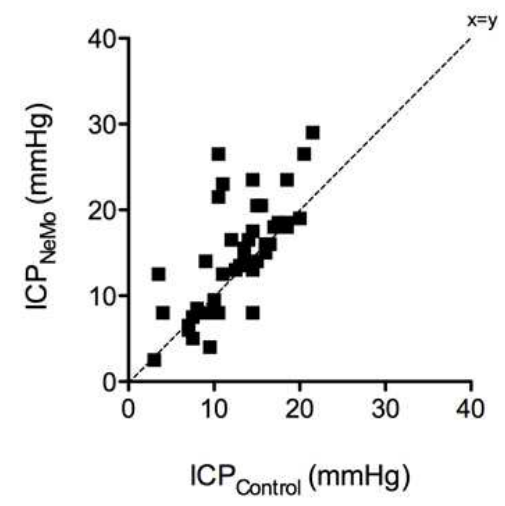

C

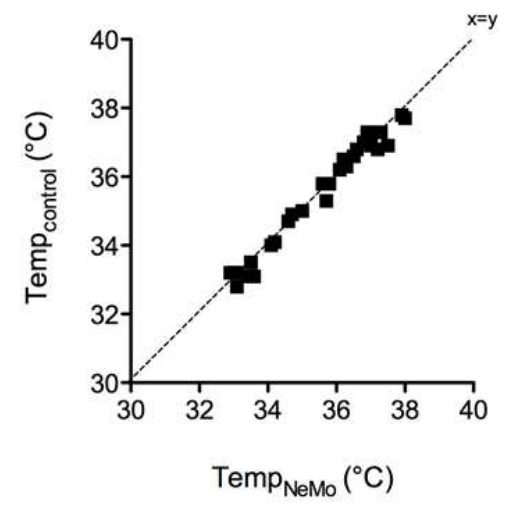

B

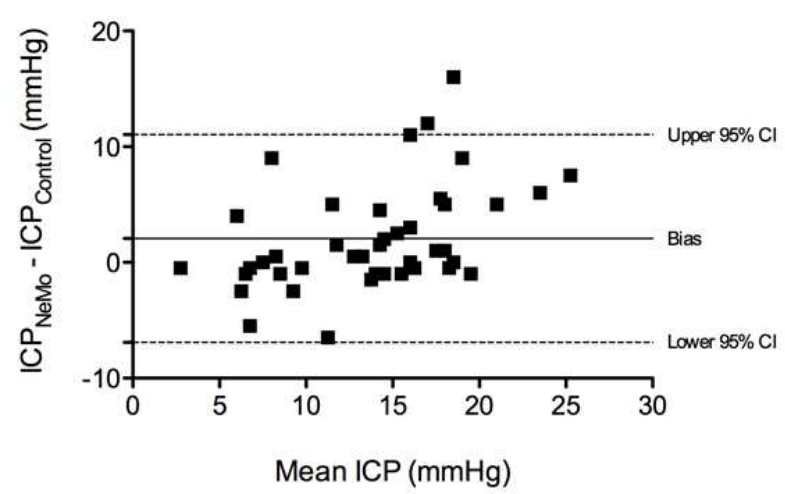

D

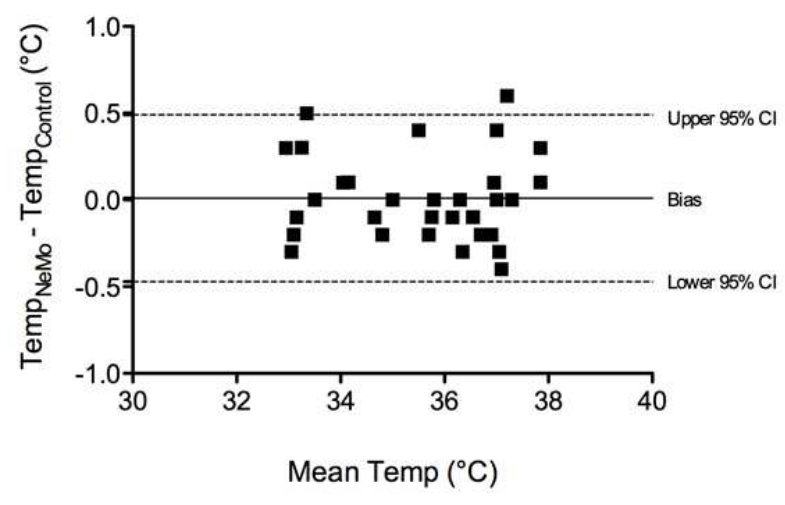


Figure 3: The ability to detect DCI from 122 NIRS-ICG measurements. Receiver operating curves for cerebral blood flow (CBF), cerebral blood volume (CBV) and mean transit time of indocyanine green (mttICG) obtained by the NeMo® Probe. Data pairs of true positive and false positive rates corresponding to each possible diagnostic threshold were plotted. Arrows mark the chosen cutoff value. Dashed lines indicate the line where the area under the curve equals 0.5 .

CBF

(ml/100g/min)

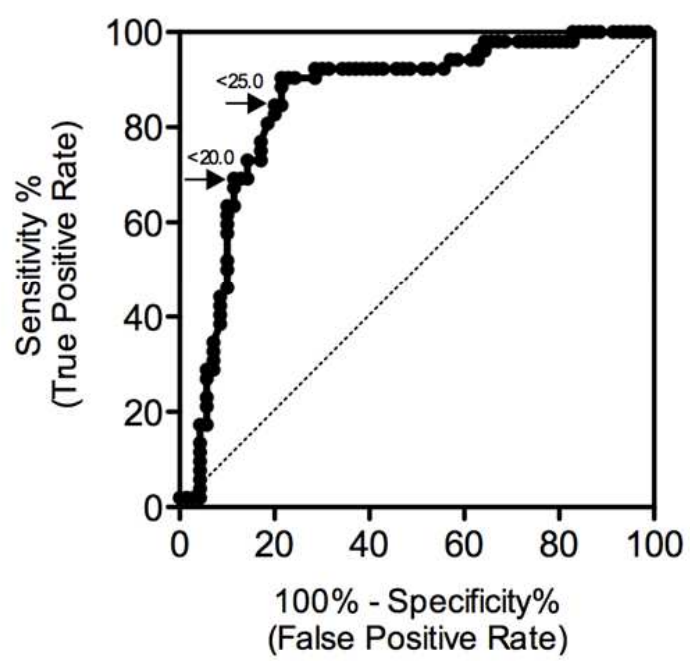

CBV

(ml/100g)

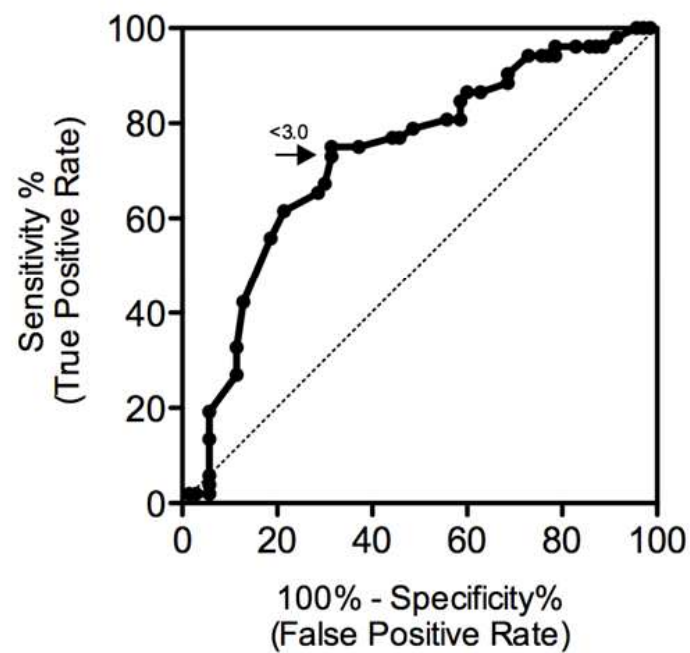

mttICG

(seconds)

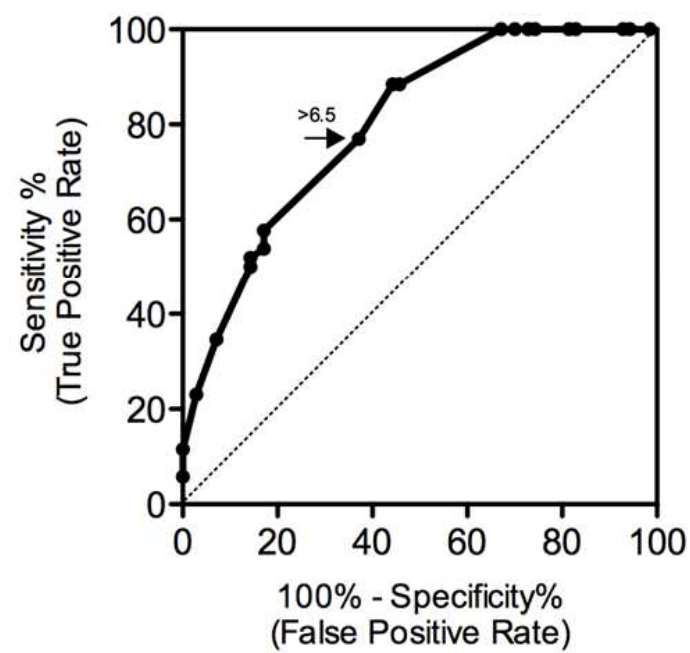


Table 1: Mean values, standard deviation and coefficient of variation from 59-pairs of repeated NIRS-ICG measurements.

\begin{tabular}{lcc}
\hline \hline & $\begin{array}{c}\text { CBF } \\
(\mathbf{m l} / \mathbf{1 0 0 g} / \mathbf{m i n})\end{array}$ & $\begin{array}{c}\text { CBV } \\
(\mathbf{m l} / \mathbf{1 0 0 g})\end{array}$ \\
\hline $\begin{array}{l}\text { Mean } \\
(\mathrm{n}=118)\end{array}$ & 30.1 & 3.2 \\
$\begin{array}{l}\mathrm{SD} \\
(\mathrm{n}=118)\end{array}$ & 12.9 & \\
$\begin{array}{l}\mathrm{CV} \\
(\mathrm{n}=59)\end{array}$ & $10.9 \%$ & 1.0 \\
\hline
\end{tabular}

$\mathrm{CBF}$, cerebral blood flow; CBV, cerebral blood volume; CV, coefficient of variation; mttICG, mean transit time of indocyanine green; SD, standard deviation 
Table 2: Measurement values from 122 NIRS-ICG measurements with and without delayed cerebral ischemia. Values are expressed as mean \pm standard deviation.

\begin{tabular}{lcc}
\hline \hline & $\begin{array}{c}\mathbf{C B F} \\
(\mathbf{m l} / \mathbf{1 0 0 g} / \mathbf{m i n})\end{array}$ & $\begin{array}{c}\mathbf{C B V} \\
(\mathbf{m l} / \mathbf{1 0 0 g})\end{array}$ \\
\hline $\begin{array}{l}\text { Values without DCI } \\
(\mathrm{n}=70)\end{array}$ & $36.8 \pm 13.8$ & $3.6 \pm 1.3$ \\
$\begin{array}{l}\text { Values with DCI } \\
(\mathrm{n}=52)\end{array}$ & $21.6 \pm 6.9$ & $2.8 \pm 0.9$ \\
$\mathrm{p}$-value & $<0.0001$ & $<0.0001$ \\
\hline
\end{tabular}

$\mathrm{CBF}$, cerebral blood flow; CBV, cerebral blood volume; DCI, delayed cerebral ischemia; mttICG, mean transit time of indocyanine green 
Table 3: Correlation between delayed cerebral ischemia and cut-off values from 128 NIRS-ICG measurements.

\begin{tabular}{|c|c|c|c|c|}
\hline Cutoff Value & $\begin{array}{l}\text { Area under the } \\
\text { curve }\end{array}$ & $\begin{array}{c}95 \% \\
\text { confidence interval }\end{array}$ & Sensitivity & Specificity \\
\hline CBF & 0.853 & $0.782-0.925$ & & \\
\hline$<20.0 \mathrm{ml} / 100 \mathrm{~g} / \mathrm{min}$ & & & $63 \%$ & $90 \%$ \\
\hline$<25.0 \mathrm{ml} / 100 \mathrm{~g} / \mathrm{min}$ & & & $85 \%$ & $83 \%$ \\
\hline CBV & 0.729 & $0.638-0.821$ & & \\
\hline$<3.0 \mathrm{ml} / 100 \mathrm{~g}$ & & & $73 \%$ & $69 \%$ \\
\hline mttICG & 0.800 & $0.723-0.876$ & & \\
\hline$>6,5 \mathrm{sec}$ & & & $77 \%$ & $63 \%$ \\
\hline
\end{tabular}

$\mathrm{CBF}$, cerebral blood flow; $\mathrm{CBV}$, cerebral blood volume; mttICG, mean transit time of indocyanine green 